\title{
Stability of an optical vortex in a circular nematic cell
}

\author{
Antonmaria A. Minzoni, ${ }^{1}$ Noel F. Smyth, ${ }^{2}$ and Zhiyong $\mathrm{Xu}^{3, *}$ \\ ${ }^{1}$ Fenomenos Nonlineales y Mecánica (FENOMEC), Departamento de Matemáticas y Mecánica, Instituto de Investigacion en Matemáticas \\ Aplicadas y Sistemas, Universidad Nacional Autónoma de México, 01000 México D.F., México \\ ${ }^{2}$ School of Mathematics and Maxwell Institute for Mathematical Sciences, University of Edinburgh, Edinburgh EH9 3JZ, \\ Scotland, United Kingdom \\ ${ }^{3}$ Nonlinear Physics Center, Research School of Physics and Engineering, Australian National University, Canberra ACT 0200, Australia
}

(Received 20 November 2009; published 10 March 2010)

\begin{abstract}
The stability of an optical vortex in a cell with a circular cross section containing a nematic liquid crystal is studied. A modulation theory based on an averaged Lagrangian formulation is developed to study this stability. It is found that the vortex is stable unless the radius of the cell is very small, nearly the width of the vortex itself. Based on the analysis of a stationary vortex, the stability of a low-amplitude vortex in a large cell under the influence of its orbital angular momentum and the repelling effect of the cell boundary are studied. The predictions of this modulation theory are found to be in excellent agreement with numerical simulations.
\end{abstract}

DOI: $10.1103 /$ PhysRevA.81.033816

PACS number(s): 42.65.Tg, 42.70.Df, 05.45.Yv

\section{INTRODUCTION}

Many optical materials exhibit a nonlinear, nonlocal response in that the response of the medium to the optical perturbation extends far beyond the waist of the beam. Examples of such media are liquid crystals [1-3] and thermal nonlinear media [4-9]. In common with other nonlinear media, self-focusing can result in the formation of self-guided optical beams due to a balance between nonlinearity and diffraction. Such self-guided beams can take the form of solitary waves [1-9] and optical vortices or vortex solitons [8,10-12]. Vortex solitons are unstable in local media, breaking up into solitary waves [13]. However, if the degree of nonlocality is large enough, optical vortices can be stable in nonlocal, nonlinear media $[8,10,12]$.

In the present work the stability of a unit-charge optical vortex soliton in a circular cell containing a nematic liquid crystal is considered. It is assumed that the cell has been rubbed to preorient the nematic to overcome the Freédericksz threshold. In previous work on the stability of a vortex soliton in a nematic liquid crystal, the Freédericksz threshold was overcome by using a static electric field to pretilt the nematic [12,14]. It was found, through a numerical linear stability analysis [12] and through an averaged Lagrangian approach [14], that the vortex is stable above a critical value of the nonlocality which depends on the amplitude of the vortex. The stability analysis based on the averaged Lagrangian approach was extended to consider the interaction of vector vortex solitons in nematic liquid crystals, the vector vortex consisting of a solitary wave in one wavelength (color) and a vortex in another wavelength (color) $[15,16]$. It was found that the solitary wave had a stabilizing effect on the vortex, so that it was stable for much lower values of nonlocality than an isolated vortex. This averaged Lagrangian approach is used to study the stability of a vortex soliton in a circular nematic cell without a pretilting field. An explicit, simple stability criterion is found which shows that vortices are stable for cell radii down to those of the order of the vortex width. This stability

\footnotetext{
*xzy124@physics.anu.edu.au
}

result is confirmed by numerical solutions. The equations for optical beams in nematic cells without a pretilting field are the same as those for thermal nonlinear media $[1,2,10]$. A numerical linear stability analysis has shown that vortex solitons of unit charge are stable in such thermal media [10]. However, this analysis was for a unit value of the nonlocality in the present nondimensionalization of the governing equations. The present analytical analysis is for arbitrary nonlocality and provides an explicit, analytical stability criterion. In Sec. III the motion of a vortex of small radius relative to that of the cell is studied.

\section{MODULATION EQUATIONS}

Let us consider the propagation of a coherent, polarized light beam through a cell filled with a nematic liquid crystal. Let us assume that the cell has a circular cross section with radius $R$ and use standard cylindrical polar coordinates $(r, \theta, z)$, with $r$ and $\theta$ the polar radius and angle in the circular cross-section. The cell is "rubbed" so that the nematic director makes an angle $\hat{\phi}$ to the $z$ direction. The angle $\phi$ is then the additional rotation of the director due to the light beam. The dimensionless equations for the field envelope $E(r, \theta, z)$ and the director deviation $\phi(r, \theta, z)$ can be written in the form [2]

$$
\begin{gathered}
i \frac{\partial E}{\partial z}+\frac{1}{2} \nabla^{2} E+\phi E=0, \\
v \nabla^{2} \phi=-|E|^{2} .
\end{gathered}
$$

Here $z$ is the direction of light propagation, $\nabla^{2}$ is the transverse (to $z$ ) Laplacian and $v$ is the nonlocality parameter, measuring the elastic response of the nematic. As the nematic molecules are fixed at the cell wall, $\phi=0$ at the cell wall, $r=R$. The appropriate boundary conditions for the electric field and nematic depend on the nature of the cell wall and the nematic anchoring conditions. However, as the electric field of the vortex decays exponentially away from its center, we make the approximation that $E=0$ at the cell wall. This assumption relies on the radius of the vortex being much less than the radius of the nematic cell. For experimental 
studies of circular vortices in circular cells, typical vortex radii are $3 \mu \mathrm{m}$ in a cell of radius $100 \mu \mathrm{m}$ for light of wavelength $532 \mathrm{~nm}$ [17]. Assuming that the radius of the vortex is much less than the radius of the cell greatly simplifies the subsequent mathematical analysis. In the evaluation of various integrals involving $E$ the range of integration was extended to infinity, as the resulting error is exponentially small. Under this approximation it is required that $E=0$ at infinity. The nonlinear system of Eqs. (1) and (2) has the Lagrangian

$$
\mathcal{L}=\int_{0}^{2 \pi} \int_{0}^{\infty} L d r d \theta
$$

where

$$
L=i r\left(E^{*} E_{z}-E E_{z}^{*}\right)-r|\nabla E|^{2}+2 r \phi|E|^{2}-v r|\nabla \phi|^{2},
$$

with an asterisk denoting the complex conjugate.

To study the dynamics of the vortex and its stability analytically, we employ the variational approach of Minzoni et al. [14] and use a trial function for the vortex soliton of the form

$$
E(r, \theta, z)=a r e^{-r / w} e^{i \sigma+i \theta}+i g(r, \theta, z) e^{i \sigma+i \theta} .
$$

The first term in Eq. (5) is the vortex soliton and the second term accounts for the low-frequency diffractive radiation generated by the internal oscillations of the vortex and that accumulates under it $[12,18]$. While the existence of this shelf of radiation has been shown from perturbation theory $[18,19]$, the reason for this radiation can most easily be seen from the dispersion relation for the linear electric field equation, $\omega=k^{2} / 2$, where $k$ is the wave number. The group velocity is then $c_{g}=k$, so that low-wave-number radiation has a low velocity and thus accumulates under the vortex, resulting in a shelf of radiation, or a pedestal, as this radiation effect is referred to in optical fibers [20,21]. Also, in analogy with previous studies of nonlocal vortices [14-16], the parameters $a, w, g$, and $\sigma$ should depend not only on the space variable $z$, but also on the azimuthal angle $\theta$.

In previous work on the evolution of beams in finite nematic cells the trial function (5) was substituted into the director equation (2) and an exact solution was found for the director distribution [22]. While an analogous solution for $\phi$ can be found using (5), unfortunately some integrals involving $\phi$ in the calculation of the averaged Lagrangian using (4) cannot be evaluated. However, when the nonlocality parameter $v$ is large the spatial scale of the optical axis distortion is much larger than the radius of the vortex [14]. Moreover, the optical axis distribution is practically flat inside the vortex core and slowly decays away from it. With these observations it was shown that the source of the optical axis distribution can be approximated by a point source distribution centered at the peak of the vortex and with the same power [14]. Comparisons with full numerical solutions below justify this point source approximation. Using this approximation the director equation (2) becomes, for large $v$,

$$
\frac{v}{r} \frac{\partial}{\partial r}\left(r \frac{\partial \phi}{\partial r}\right)=-\frac{a^{2} w^{3}}{4} \delta(r-w) .
$$

This equation has the solution

$$
\phi= \begin{cases}\frac{a^{2} w^{4}}{4 v} \ln (R / w), & r<w \\ \frac{a^{2} w^{4}}{4 v} \ln (R / r), & w<r \leqslant R,\end{cases}
$$

which will be shown to give a good approximation to the director distribution.

Earlier work on vortex stability has shown that to determine linear stability, only the special class of perturbations about the fixed point which have $\sigma$ depending on $z$ needs to be considered [14-16]. With this restriction, substituting the trial function (5) and the director solution (7) into the Lagrangian (4), then integrating with respect to $r$, we obtain an averaged Lagrangian for the modulation parameters $a, w, g$, and $\sigma$. This averaged Lagrangian is

$$
\begin{aligned}
\mathcal{L}= & -\left(\frac{3}{4} a^{2} w^{4}+2 \Lambda_{1} g^{2}\right) \frac{\partial \sigma}{\partial z}-4 a w^{3} \frac{\partial g}{\partial z}+4 g w^{3} \frac{\partial a}{\partial z} \\
& +12 a g w^{2} \frac{\partial w}{\partial z}-\frac{3}{8} w^{2} a^{2}-\frac{1}{4} w^{2}\left(\frac{\partial a}{\partial \theta}\right)^{2} \\
& -\frac{1}{2} a w \frac{\partial a}{\partial \theta} \frac{\partial w}{\partial \theta}-\frac{3}{8} a^{2}\left(\frac{\partial w}{\partial \theta}\right)^{2}+2 w g \frac{\partial a}{\partial \theta} \\
& +2 a g \frac{\partial w}{\partial \theta}-2 a w \frac{\partial g}{\partial \theta}-\Lambda_{2}\left[\left(\frac{\partial g}{\partial \theta}\right)^{2}+g^{2}\right] \\
& +\frac{a^{4} w^{8}}{8 v} \ln (R / w)-\frac{a^{4} w^{8}}{2 v} I_{1} \\
& +\frac{a^{2} w^{6} g^{2}}{16 v}[5 \ln (R / w)+5-9 \ln (3 / 2)],
\end{aligned}
$$

on using the assumption discussed previously that the radius of the vortex is much smaller than the radius of the nematic cell. Here

$$
I_{1}=\int_{1}^{\infty} y^{3} \ln (y) e^{-2 y} d y .
$$

The terms $\Lambda_{1}$ and $\Lambda_{2}$ in Eq. (8) describe the effect of the shelf of radiation [18]. This radiation is assumed to be radially symmetric in space, centered about the vortex peak at $r=w$, and of width $R_{s}$, as follows from numerical solutions. Hence $g$ is only nonzero in the region $r_{\min }<r<r_{\max }$, where $r_{\text {min } \max }=w \mp R_{s} / 2$. Then

$$
\Lambda_{1}=w R_{s} \quad \text { and } \quad \Lambda_{2}=\ln \left(\frac{r_{\max }}{r_{\min }}\right) .
$$

In previous work it was found that $R_{s}=w$ gives a good approximation to the width of the shelf of radiation [14].

The steady vortex can be found from the averaged Lagrangian (8) as a fixed point by setting the derivatives of $a$, $w$, and $g$ to be 0 , with $\sigma_{z}$ taken to be constant. Denoting fixed point values with the subscript 0 , it can be found that the steady vortex is determined by

$$
6 v=a_{0}^{2} w_{0}^{6}, \quad \sigma_{0 z}=\frac{2}{w_{0}^{2}} \ln \left(R / w_{0}\right),
$$

in the limit $R \gg w_{0}$. 
The modulation equations for the vortex evolution are obtained from variations of the averaged Lagrangian (8) with respect to the vortex parameters. Linear stability is then determined by perturbing around the fixed point with $a=a_{0}+a_{1}, w=w_{0}+w_{1}, g=g_{1}$, and $\sigma=\sigma_{0}(z)$, where $\left|a_{1}\right| \ll a_{0},\left|w_{1}\right| \ll w_{0}$, and $\left|g_{1}\right| \ll 1$. The linearized stability equations for the vortex are therefore

$$
\begin{aligned}
8 a_{0} w_{0}^{2} \frac{\partial w_{1}}{\partial z}= & \frac{8 \Lambda_{1}}{w_{0}^{2}} \ln \left(R / w_{0}\right) g_{1}+4 a_{0} \frac{\partial w_{1}}{\partial \theta}+2 \Lambda_{2} g_{1} \\
& -2 \Lambda_{2} \frac{\partial^{2} g_{1}}{\partial \theta^{2}}-\frac{a_{0}^{2} w_{0}^{6}}{8 v}\left[5 \ln \left(R / w_{0}\right)+5\right. \\
& -9 \ln (3 / 2)] g_{1}, \\
8 a_{0} w_{0}^{3} \frac{\partial g_{1}}{\partial z}= & -\frac{3}{2} a_{0}^{2} w_{0} w_{1}+\frac{3}{4} a_{0}^{2} w_{0} \frac{\partial^{2} w_{1}}{\partial \theta^{2}} \\
& +4 a_{0} w_{0} \frac{\partial g_{1}}{\partial \theta} \\
a_{1}= & -\frac{2 a_{0}}{w_{0}} w_{1} .
\end{aligned}
$$

We now perform a modal stability analysis on the linearized equations (12) and (13), with

$$
w_{1}=W(z) e^{i l \theta} \quad \text { and } \quad g_{1}=G(z) e^{i l \theta},
$$

and determine the stability from the resulting system of ordinary differential equations. If we set $A_{s}=a_{0} w_{0} e^{-1} / \sqrt{v}$ to be the scaled amplitude of the steady-state vortex, then the eigenvalues of these ordinary differential equations have a positive real part provided that

$$
\begin{aligned}
& \frac{e^{2} A_{s}^{2} w_{0}^{4}}{8}\left[5 \ln \left(R / w_{0}\right)+5-9 \ln (3 / 2)\right]-\frac{8 \Lambda_{1}}{w_{0}^{2}} \ln \left(R / w_{0}\right) \\
& -2 \Lambda_{2}\left(1+l^{2}\right)>0 .
\end{aligned}
$$

The vortex is then unstable if this inequality is satisfied with $l=1$. Using expressions (10) for $\Lambda_{1}$ and $\Lambda_{2}$ and the steadystate relation (11), the vortex is then unstable if

$$
17 \ln \frac{R A_{s} e}{\sqrt{6}}<15-27 \ln (3 / 2)-16 \ln 3
$$

Or

$$
R A_{s}<0.4067 \text {. }
$$

To confirm the analytical criteria (18) from modulation theory on the stability of vortex solitons in circular nematic cells and to validate the point source approximation for the calculation of the director distribution, we solve the system (1) and (2) numerically with the beam propagation method by applying the Crank-Nicholson algorithm. The main conclusion is that the vortex solitons are stable if the radius of the cell is large enough compared with the width of the vortex solitons. For example, for the case where the radius of the cell $R=40$, our results show that the vortex solitons are stable in the whole domain of their existence. An illustrative example of such a case is shown in Figs. 1(a) and 1(b), from which one can see that the vortex soliton can propagate in a stable manner over a long distance, still keeping the perfect structure as at input. Thus the circular nematic cell proves to be a good setting for the stabilization of vortex solitons. Furthermore, our results show that vortex solitons in a relatively small radius cell still can be
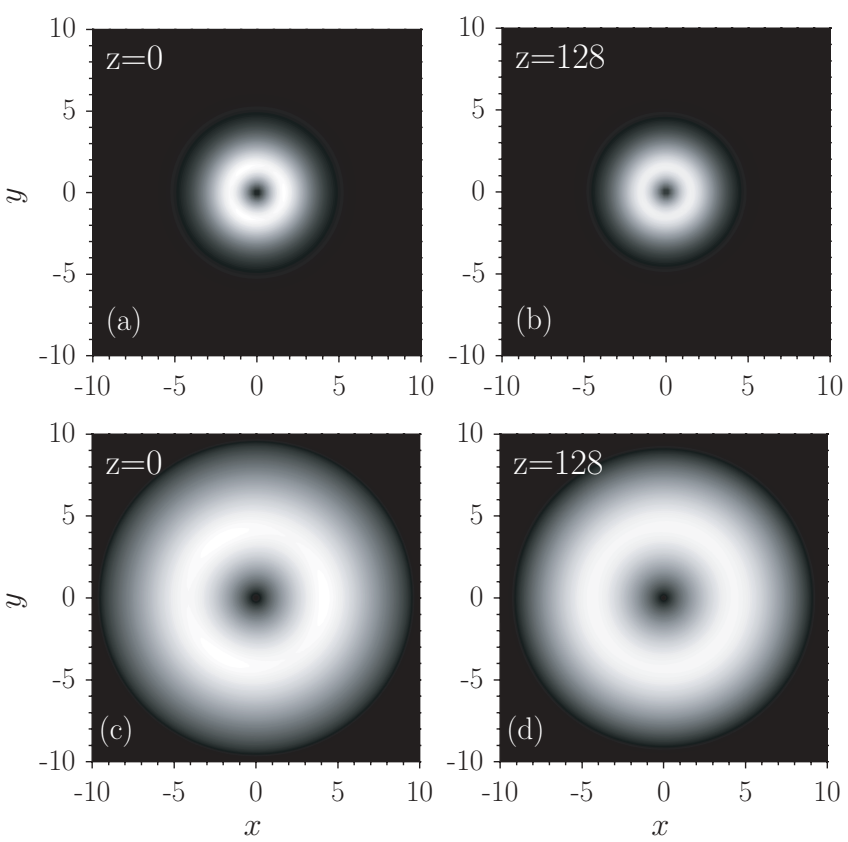

FIG. 1. Stable propagation of vortex solitons in circular nematic cells with different radii. (a, b) Radius of the cell $R=40$, and scaled amplitude of the vortex soliton $A_{s}=0.687$. (c, d) Radius of the cell $R=10$, and scaled amplitude $A_{s}=0.087$.

stabilized. For example, for a cell radius $R=10$, a vortex soliton with scaled amplitude $A_{s}=0.087$ has the product $R A_{s}=0.87$, which is still larger than the critical value 0.4067 for stability. Thus according to criterion (18), the vortex soliton should be stable, and as shown in Figs. 1(c) and 1(d), the vortex soliton is indeed stable during propagation. However, it should be pointed out that the numerical test works well down to the product $R A_{s}=0.87$, and it is difficult to test below that numerically. Overall our results show excellent agreement between numerical simulations and the analytical criterion from the modulation theory. It is noted that the vortices do not diffract, as their power is large enough that there is a balance between linear diffraction and nonlinear self-focusing, resulting in a coherent structure [17].

\section{MOTION OF A SMALL VORTEX IN A LARGE CELL}

The motion of a vortex in a large, finite, circular cell under the influence of its angular momentum and the repelling effect of the bounding wall is now studied using the technique of Minzoni et al. [14] for an infinite cell. The elliptic equation (2) for the director has a simple, closed-form Green's function, as it is just Poisson's equation. In Cartesian coordinates the Lagrangian for the nematicon equations (1) and (2) to be averaged is then

$$
\begin{aligned}
L= & i\left(E^{*} E_{z}-E E_{z}^{*}\right)-|\nabla E|^{2} \\
& +|E|^{2} \iint_{\Omega} G\left(\left|\mathbf{x}-\mathbf{x}^{\prime}\right|\right)\left|E\left(\mathbf{x}^{\prime}\right)\right|^{2} d \mathbf{x}^{\prime},
\end{aligned}
$$


where $\Omega$ is the circular cell region $\left|\mathbf{x}^{\prime}\right|=R$. The Green's function is obtained using the method of images and is

$$
G\left(\left|\mathbf{x}-\mathbf{x}^{\prime}\right|\right)=\frac{1}{2 \pi v}\left[\ln \frac{1}{\left|\mathbf{x}-\mathbf{x}^{\prime}\right|}-\ln \frac{R}{\left.\left|\mathbf{x}^{\prime}\right|\left|\mathbf{x}-R^{2} \mathbf{x}^{\prime} /\right| \mathbf{x}^{\prime}\right|^{2} \mid}\right] .
$$

The appropriate trial function is as the trial function (5), but with $r=|\mathbf{x}-\mathbf{u}|$, so that the vortex is centered at $\mathbf{x}=\mathbf{u}$. To concentrate on the motion of the vortex, $a, w$, and $\dot{\sigma}$ are taken to be constant and the shed radiation is neglected, so that $g=0$. Previous studies have found that the amplitude and width oscillation of the beam essentially decouples from its center-of-mass motion [23-25].

With a momentum term to account for the center-of-mass motion, the appropriate trial function is

$$
E(\mathbf{x}, \mathbf{u}, z)=a r e^{-r / w} e^{i \sigma+i \theta} e^{i \mathbf{p} \cdot(\mathbf{x}-\mathbf{u}(z))}, \quad r=|\mathbf{x}-\mathbf{u}(z)| .
$$

Substitution of this trial function into the Lagrangian (19) and averaging gives the Lagrangian (8), supplemented by a linear momentum contribution and an image correction. Because $a, w$, and $\dot{\sigma}$ are assumed constant, the averaged Lagrangian is

$\mathcal{L}=-\frac{3}{4} a^{2} w^{4} \dot{\sigma}-\frac{3}{8} a^{2} w^{2}+\frac{a^{2} w^{3}}{4}\left(\mathbf{p} \cdot \dot{\mathbf{x}}-\frac{1}{2} \mathbf{p} \cdot \mathbf{p}\right)+\frac{\mathcal{L}_{v}}{2 \pi}$,

where $\mathcal{L}_{\nu}$ is the contribution of the nonlocal (Green's function) term. To evaluate this nonlocal term, we consider the translation invariant term of the Green's function. Because it is assumed that the cell is much larger than the vortex width, the integration in the nonlocal term can be extended to the whole plane. The dependence on $\mathbf{x}$ then cancels out due to translation invariance, and the contribution

$$
-\frac{a^{4} w^{8}}{2 v} I_{1}
$$

is obtained, which is expected from the averaged Lagrangian (8). The contribution due to the image point is

$$
\begin{aligned}
\mathcal{L}_{v}= & \frac{1}{2 \pi v} \int_{-\infty}^{\infty} \int_{-\infty}^{\infty} \int_{-\infty}^{\infty} \int_{-\infty}^{\infty}|E(\mathbf{x}-\mathbf{u})|^{2} \\
& \times \ln \left(\frac{\left|\mathbf{x}^{\prime}\right|}{R}\left|\mathbf{x}-\frac{R \mathbf{x}^{\prime}}{\left|\mathbf{x}^{\prime}\right|^{2}}\right|\right)\left|E\left(\mathbf{x}^{\prime}-\mathbf{u}\right)\right|^{2} d \mathbf{x}^{\prime} d \mathbf{x}
\end{aligned}
$$

To evaluate this integral we change to polar coordinates centered at $\mathbf{x}^{\prime}=\mathbf{u}$ and $\mathbf{x}=\mathbf{u}$. Then as $|E|^{2}$ is approximated by a $\delta$ function centered at the vortex radius, the radial integrals can be readily determined by evaluating the Green's function at the appropriate singularities of the $\delta$ functions. The angular integrals are evaluated by expanding in a Taylor series about $w=0$, since the vortex is assumed to be small relative to the radius of the cell. The first-order angular contribution is 0 and the first nonzero contribution is the $O\left(w^{2}\right)$ term,

$$
\mathcal{L}_{v}=\frac{2 \pi}{v} \frac{a^{4} w^{8}}{8} \ln \left|\frac{R^{2}-|\mathbf{x}(z)|^{2}}{R w}\right| .
$$

The final averaged Lagrangian is then

$$
\begin{aligned}
\mathcal{L}= & -\frac{3}{4} a^{2} w^{4} \dot{\sigma}-\frac{3}{8} a^{2} w^{2}+\frac{a^{2} w^{3}}{4}\left(\mathbf{p} \cdot \dot{\mathbf{x}}-\frac{1}{2} \mathbf{p} \cdot \mathbf{p}\right) \\
& +\frac{a^{4} w^{8}}{8 v} \ln \left|\frac{R^{2}-|\mathbf{x}(z)|^{2}}{R w}\right|-\frac{a^{4} w^{8}}{2 v} I_{1} .
\end{aligned}
$$

Variations of the averaged Lagrangian (26) with respect to $a$ and $w$ when $\mathbf{u}=\mathbf{0}$ recover the fixed point relation (11). Variations with respect to $\mathbf{u}$ and $\mathbf{p}$ give the equations of motion for the center of mass of the vortex. To begin with, let us assume that the vortex has no angular momentum, so that $\mathbf{u}=(u(z), 0)$ and $\mathbf{p}=(p(z), 0)$. Then the center-of-mass variational equations are

$$
\dot{x}=p \quad \text { and } \quad \dot{p}+\frac{a^{2} w^{5}}{v\left(R^{2}-x^{2}\right)} x=0
$$

or

$$
\ddot{x}+\frac{a^{2} w^{5}}{v} \frac{x}{R^{2}-x^{2}}=0 .
$$

The motion of the center of mass of the vortex is then just an oscillatory motion around the center of the cell due to the repulsive effect of the walls, as for the case of a rectangular cell [22].

The general case for which the vortex has angular momentum can be treated similarly. We introduce polar coordinates based on the center of the cell, where $|\mathbf{u}|=\eta$ and $\varphi$ is the corresponding polar angle. The resulting equations are similar to those for the Kepler problem with a repulsive potential due to the walls [26] and are

$$
\frac{d}{d z} \eta^{2} \dot{\varphi}=0, \quad \dot{\varphi}=\frac{L}{\eta^{2}}, \quad \ddot{\eta}-\frac{L^{2}}{\eta^{3}}+\frac{a^{2} w^{5}}{v} \frac{\eta}{R^{2}-\eta^{2}}=0,
$$

where $L$ is the conserved angular momentum. This equation has only a periodic solution for $\eta$, since the center of the cell is repulsive due to the angular momentum, as well as the cell walls being repulsive. The solution for $\eta$ oscillates about the fixed point

$$
\eta_{f}^{2}=\frac{v}{2 a^{4} w^{5}}\left[L^{2}+\sqrt{L^{4}+\frac{4 a^{4} w^{5} R^{2}}{v}}\right] .
$$

For large $R$ this takes the form

$$
\eta_{f}=\frac{v^{1 / 4} R^{1 / 2}}{a w^{5 / 4}} .
$$

The vortex then orbits around this center as it advances slowly down the cell with $\dot{\varphi} \propto L / R$. The vortex will eventually settle into a uniform circular motion on a circle of radius $\eta_{f}$ with angular velocity $L / \eta_{f}^{2}$.

The motion of the vortex to leading order is then simple due to the circular symmetry of the cell. However, as higher-order terms are included in the expansion for the nonlocal term, the angular momentum is no longer conserved and the system could, in principle, move along very complicated trajectories around the center $\eta_{f}$. 


\section{CONCLUSIONS}

We have studied optical vortex solitons propagating in a cell with a circular cross section containing a nematic liquid crystal. In particular, we developed the simple modulation theory to provide analytical criteria for the stability of vortex solitons. Our results show that the vortex solitons are stable unless the radius of the cell is very small, nearly the width of the vortex itself. The simple modulation theory has been found to give predictions in excellent agreement with numerical simulations. This modulation theory has been extended to study the motion of a vortex not in the center of the cell. Due to the repulsion effect of the cell wall, the vortex was found to orbit about the center of the cell.

\section{ACKNOWLEDGMENTS}

The authors thank Professor Y. S. Kivshar for many helpful discussions related to this work. One of the authors $(\mathrm{Z} . \mathrm{Xu})$ would like to thank Yaroslav Kartashov for useful discussions. This research was supported by the Royal Society of London, under Grant No. JP090179, and the Australian Research Council.
[1] G. Assanto, M. Peccianti, and C. Conti, Opt. \& Photon. News 14, 45 (2003).

[2] C. Conti, M. Peccianti, and G. Assanto, Phys. Rev. Lett. 91, 073901 (2003).

[3] C. Conti, M. Peccianti, and G. Assanto, Phys. Rev. Lett. 92, 113902 (2004)

[4] C. Rotschild, B. Alfassi, O. Cohen, and M. Segev, Nature Phys. 2, 769 (2006).

[5] C. Rotschild, T. Schwartz, O. Cohen, and M. Segev, Nature Photonics 2, 371 (2008).

[6] C. Rotschild, M. Segev, Z. Xu, Y. V. Kartashov, L. Torner, and O. Cohen, Opt. Lett. 31, 3312 (2006).

[7] B. Alfassi, C. Rotschild, O. Manela, M. Segev, and D. N. Christodoulides, Opt. Lett. 32, 154 (2007).

[8] C. Rotschild, O. Cohen, O. Manela, M. Segev, and T. Carmon, Phys. Rev. Lett. 95, 213904 (2005).

[9] B. Alfassi, C. Rotschild, O. Manela, M. Segev, and D. N. Christodoulides, Phys. Rev. Lett. 98, 213901 (2007).

[10] Y. V. Kartashov, V. A. Vysloukh, and L. Torner, Opt. Express 15, 9378 (2007).

[11] Y. V. Kartashov, V. A. Vysloukh, and L. Torner, Opt. Express 15, 16216 (2007).

[12] A. I. Yakimenko, Yu. A. Zaliznyak, and Yu. S. Kivshar, Phys. Rev. E 71, 065603(R) (2005).
[13] A. S. Desyatnikov, Yu. S. Kivshar, and L. Torner, in Progress in Optics, edited by E. Wolf (North-Holland, Amsterdam, 2005), Vol. 47.

[14] A. A. Minzoni, N. F. Smyth, A. L. Worthy, and Y. S. Kivshar, Phys. Rev. A 76, 063803 (2007).

[15] A. A. Minzoni, N. F. Smyth, Z. Xu, and Y. S. Kivshar, Phys. Rev. A 79, 063808 (2009).

[16] Z. Xu, N. F. Smyth, A. A. Minzoni, and Y. S. Kivshar, Opt. Lett. 34, 1414 (2009).

[17] Y. Izdebskaya, private communication, Nonlinear Physics Centre, Australian National University.

[18] W. L. Kath and N. F. Smyth, Phys. Rev. E 51, 1484 (1995).

[19] J. Yang, Stud. Appl. Math. 98, 61 (1997).

[20] T. Jintao, L. Hongjun, G. Cunxiao, Z. Wei, W. Yishan, and C. Guofu, Opt. Commun. 282, 74 (2009).

[21] Q. Li, K. Senthilnathan, K. Nakkeeran, and P. K. A. Wai, J. Opt. Soc. Am. B 26, 432 (2009).

[22] A. Alberucci, G. Assanto, D. Buccoliero, A. S. Desyatnikov, T. R. Marchant, and N. F. Smyth, Phys. Rev. A 79, 043816 (2009).

[23] C. García-Reimbert, A. A. Minzoni, T. R. Marchant, N. F. Smyth, and A. L. Worthy, Physica D 237, 1088 (2008).

[24] B. D. Skuse and N. F. Smyth, Phys. Rev. A 77, 013817 (2008).

[25] B. D. Skuse and N. F. Smyth, Phys. Rev. A 79, 063806 (2009).

[26] G. Assanto, N. F. Smyth, and A. L. Worthy, Phys. Rev. A 78, 013832 (2008). 